\title{
Sensitivity of Age Estimation Systems to Demographic Factors and Image Quality: Achievements and Challenges
}

\author{
Ali Akbari, Muhammad Awais and Josef Kittler \\ Centre for Vision, Speech and Signal Processing (CVSSP) \\ University of Surrey, Guildford, UK \\ \{ali.akbari,m.a.rana,j.kittler\}@surrey.ac.uk
}

\begin{abstract}
Recently, impressively growing efforts have been devoted to the challenging task of facial age estimation. The improvements in performance achieved by new algorithms are measured on several benchmarking test databases with different characteristics to check on consistency. While this is a valuable methodology in itself, a significant issue in the most age estimation related studies is that the reported results lack an assessment of intrinsic system uncertainty. Hence, a more in-depth view is required to examine the robustness of age estimation systems in different scenarios. The purpose of this paper is to conduct an evaluative and comparative analysis of different age estimation systems to identify trends, as well as the points of their critical vulnerability. In particular, we investigate four age estimation systems, including the online Microsoft service, two best state-of-the-art approaches advocated in the literature, as well as a novel age estimation algorithm. We analyse the effect of different internal and external factors, including gender, ethnicity, expression, makeup, illumination conditions, quality and resolution of the face images, on the performance of these age estimation systems. The goal of this sensitivity analysis is to provide the biometrics community with the insight and understanding of the critical subject-, camera- and environmental-based factors that affect the overall performance of the age estimation system under study.
\end{abstract}

\section{Introduction}

Age estimation aims to find a function that maps a face image to its corresponding age label. This is a very challenging task. A number of demographic factors including ethnicity, gender and gene, as well as dependence on external factors such as expression, image quality, illumination

b978-1-7281-9186-7/20/\$31.00 (c)2020 Crown conditions, makeup, etc have introduced challenges to the age estimation problem $[22,11]$.

In order to have a deep insight of the performance of an age estimation system, we need to perform both sensitivity and optimisation analysis. A sensitivity analysis is the study of how the output of an age estimation system is affected by different factors, while optimisation analysis is the determination of system design and parameter settings that yield the best system performance [19]. A significant issue in the most age estimation related studies, and also in other machine learning based applications [1, 2, 3, 4, 5, 6, 8, 9], is that they focus on the system optimisation and the reported results in these studies are usually concerned with the overall accuracy of the system assessed in terms of some accuracy metrics. Such studies neglect the critical questions of sensitivity of the results to different factors. The aim of this paper is to address this deficiency by conducting an effective and insightful analysis of the sensitivity of existing age estimation systems to gain understanding of the key critical factors which affect their behaviour and overall performance. A further motivation is to provide an indication of their vulnerability and identify the scope for their improvement.

To the best of our knowledge, there are few works [11] which assess the performance of age estimation to some intrinsic factors, including gender, ethnicity, expression. However, none on these works analyse the effect of cameraand environmental-based extrinsic factors, such as illumination conditions, image quality and resolution on the age prediction accuracy. In addition, these studies analyse the sensitivity of the system on a small database under a closedset scenario where the training and test sets come from the same database. Such closed-set evaluation fails to provide fully comprehensive understanding of automatic age estimation methods.

In this paper, we perform an empirical evaluation of different facial age estimation solutions, with participation from one online age estimation service and the two best state-of-the-art approaches, over nine target databases. We 
also present a novel age estimation system by proposing a loss function for training the model. We focus on deep endto-end architectures for the age estimation problem, and use a cross-dataset evaluation where the training and test sets have different characteristics. The age estimation performance of these systems is then assessed demographically by gender and ethnicity. The impact of image-related variations, namely image quality, resolution, illumination conditions, expression and makeup is also documented. The proposed system provides better results under the cross dataset evaluation, compared with the other adopted systems. To the best of our knowledge, this is the first work that performs a comprehensive analysis of the sensitivity of the state-of-the-art age estimation systems to the changes in facial features caused by the above-mentioned factors.

\section{The Frameworks}

In this section, we review four different approaches to the age estimation problem.

\subsection{Commercial online age estimation services}

There are several on-line age estimators developed by experienced companies. As reported in [7], the Microsoft's model, which uses a multi-layered deep learning methodology, achieves the most satisfactory performance among all existing online services.

\subsection{Academic State-of-the art approaches}

As for many pattern recognition problems, age estimation can be achieved by different approaches. We consider two recent deep age estimation systems, reporting the best performance in the literature.

\section{Classification based approaches}

A typical age estimation algorithm can be developed by modelling the problem as a general classification paradigm with a number of $K$ classes [28] bu using the cross entropy (CE) as the loss function. The major issue with this approach is that it assumes that the ages are considered as independent classes. Consequently, the order relationship among the labels is ignored during training. In order to alleviate this issue, Pan et al. [24] proposed to integrate the $\mathrm{CE}$ loss function with regression terms designed to manage the order relationship among labels. This loss, named as CE-mean-variance (CE-MV) loss, is defined as $L_{C E-M V}(\mathbf{p}, y)=L_{C E}+\lambda_{1}\left(\mu_{p}-y\right)^{2}+\lambda_{2} \sigma_{p}^{2}$, where $\lambda_{1}$ and $\lambda_{2}$ are regularisation parameters. $\mu_{p}$ and $\sigma_{p}^{2}$ are the mean and the variance of the model's output $\mathbf{p}$, respectively.

https://www.microsoft.com/cognitive-services/ en-us/face-api/

\section{Label distribution based approaches}

In addition to the order relationship among labels, there exist strong semantic correlations among close age labels which leads to visual label ambiguity [15]. It is evident that the problem formulation should implicitly or explicitly take into account this label ambiguity during training stage. This can be achieved using label distribution learning, proposed in [15]. In this approach, a probability distribution (Gaussian distribution), centred on the ground-truth age label $y$, is assigned to each subject. In this type of age label encoding, the $k$-th element represents the degree (probability) to which the face instance belongs to the age label $k$, considering the semantic correlation of the adjacent ages.

With this formulation, the age estimation problem is converted into a distribution learning problem. Here, the goal is to train a deep network to find a mapping function between the input face image $\mathbf{x}$ and its corresponding label distribution q. Assume $p_{k}$ and $q_{k}$ are the $k$-th entries of the estimated and ground-truth distributions, i.e. $\mathbf{p}$ and $\mathbf{q}$, respectively. The Kullback-Leibler (KL) divergence defined as:

$$
L_{K L}(\mathbf{p}, \mathbf{q})=\sum_{k=1}^{K} q_{k} \log \left(\frac{q_{k}}{p_{k}}\right)
$$

is the most popular loss function for training a model when the age labels are encoded as label distributions $[13,16]$.

Gao et al. [14] propose the use of KL loss function regularised by an expectation regression module. This loss function, named KL-mean (KL-M), is defined as:

$$
L_{K L-M}(\mathbf{p}, \mathbf{q})=\sum_{k=1}^{K} q_{k} \log \left(\frac{q_{k}}{p_{k}}\right)+\lambda_{1}\left|\mu_{p}-y\right|
$$

to further refine the value predicted by the KL loss function. The expectation regression module penalises the difference between the mean of an estimated age distribution and the ground-truth age label.

\section{Proposed loss function}

To make a system that behaves as we expect, we have to choose a proper divergence measure as the loss function for the label distribution learning approach. One issue with the KL loss function is that it is not symmetric, i.e. $L_{K L}(\mathbf{p}, \mathbf{q}) \neq L_{K L}(\mathbf{q}, \mathbf{p})$. This asymmetric nature of the KL loss function is a crucial aspect and leads to an improper distance between two distributions. A recent study [30] shows that symmetric losses are robust to noisy labels which is a common issue in the age estimation datasets. We propose to use the Matusita's distance (MD) [10] which addresses the asymmetry issue of the KL 
divergence. The proposed MD loss function is defined as:

$$
L_{M D}(\mathbf{p}, \mathbf{q})=\frac{1}{2} \sum_{k=1}^{K}\left(\sqrt{p_{k}}-\sqrt{q}\right)^{2} .
$$

\section{Experimental Settings}

In this section, we introduce our experimental settings. Mean absolute error (MAE) and cumulative score (CS) are used as the two metrics to evaluate the performance of age estimation. MAE is obtained by averaging the error over the test data as $\sum_{i} \frac{\left|\hat{y}_{i}-y_{i}\right|}{M}$, where $M$ is the total number of test images. The estimation accuracy is given by the CS measure, calculated as $\frac{M_{I}}{M} \times 100 \% . M_{I}$ is the number of the images whose $\left|\hat{y}_{i}-y_{i}\right|<I$. In this paper, $I$ is set as 5 .

\subsection{Training and Evaluation Databases}

We use a cleaned version of the IMDB-WIKI database [28] provided by [31] and then removed all multiperson images from the database. Due to small number of images in younger and older ages, we also crawled a new in-the-wild set of 23, 876 facial images of people with ages over 70 years old and younger than 20 years old from the Internet. Finally, we mixed these two collections with other three small ageing databases, including AgeDB [21] and UTKFace [32], respectively to build a novel cleaned ageing database, namely BAlanced Ageing (BAG) database, being a collection of 200,123 images from age 0 to 100 .

The performance is evaluated on the following databases:

MORPH database [27] is a large longitudinal collection of 55,134 images from 13, 617 subjects of different ethnicity in the age range from 16 to 77 years old. More than $96 \%$ of the images in the MORPH database are African or European people.

FG-NET database [25] contains 1,002 images of 82 subjects and the ages range from 0 to 69 years old.

FACES database [12] has 2, 052 images of 171 subjects with six expressions (neutrality, happiness, anger, fear, disgust, and sadness) in the age range from 19 to 80 years old. SC-FACE database [17] contains 4, 160 images of 130 subjects in the age range from 21 to 75 years old. We separate this database into two separate databases, namely SCFACE-ROT and SC-FACE-SUR, which contain 1, 170 and 2, 990 images, respectively. Taken by a digital high-quality camera, each subject in the SC-FACE-ROT database has one high-resolution frontal image and 9 images with different head poses ranging from -90 to +90 degrees in equal steps of 22.5 degrees. Each subject in the SC-FACE-SUR database has 17 images with different qualities.

Target-BAG (T-BAG) database is built by separating randomly 4,000 images from our BAG database.

MULTI-PIE database [18] is a subset of images of 100 subject from the original MULT-PIE database. Each subject has one high-resolution frontal image and 19 frontal images with different illumination conditions.

\subsection{Implementation Details}

We use the pre-trained VGG-Face [26] trained with a large-scale face recognition database as the basic backbone of our system. We employ the stochastic gradient descent optimiser and set the learning rate, the momentum and the weight decay as $0.001,0.9$ and 0.0005 , respectively. The learning is decreased exponentially for 30 epochs. All training and test images are aligned by the five facial landmarks using the procedure described in [20]. Random horizontal flipping, rotating and cropping are adopted as the data augmentation techniques. The central cropped image is used during inference and the maximum value over the entries of the output vector is considered as the predicted age.

\section{Sensitivity Analysis}

This section provides a comprehensive analysis of different factors influencing the age estimation performance, including gender, ethnicity, expression, head pose, makeup, resolution and quality of face images.

\subsection{Effect of Gender on Age Estimation}

The effect of gender on the age estimation accuracy is evaluated by testing each system for both males and females. For this purpose, we categorise all the images from FG-NET, MORPH and T-BAG databases according to gender and compute MAEs and CS scores for different age estimation systems with respect to each gender. From the results, shown in Table 1, our age estimation method achieves better performance compared to other methods. Note that the overall MAE for male subjects is significantly lower than females, meaning that the age estimation for females is more difficult. Consequently, more attention to this phenomenon is required in order to further enhance the overall age estimation performance in the future.

Fig. 2 illustrates the average predicted age at each age for each gender separately. The solid line indicates the average predicted age in each of the 101 age classes. The dashed line is a reference to show where accurate predictions must lie. The dot points show the expectation of the predicted age for each true age and the red curve is the fitted curve to these dot points. Since the curves of all competing systems follow the same pattern, we only plot the curve of our proposed method for better visualisation. In the case of males (Fig. 2a), the ages are consistently overestimated from ages 15 to 25 and ages 50 to 70 . While in other age intervals, one can observe a tendency to under-estimate the ages of males. Interestingly, for all females more than 20 years old, the ages tend to be underestimated, except the ages from 50 to 65 years old, where the predicted ages are close to the true line (cf., Fig. 2b). 


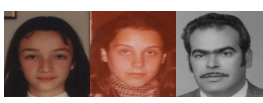

FGNET

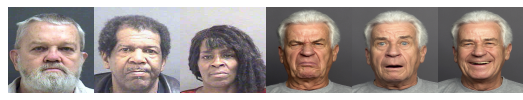

MORPH
FACES

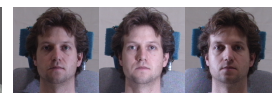

PIE

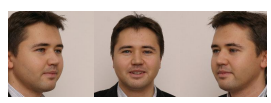

$\mathrm{SC}$

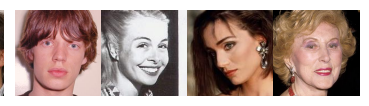

T-BAG

Figure 1. Sample images from the evaluation databases.

Table 1. MAEs and CS Scores with Respect to Gender

\begin{tabular}{|c|c|c|c|c|c|c|c|c|}
\hline & \multicolumn{2}{|c|}{ Microsoft } & \multicolumn{2}{|c|}{ CE-MV } & \multicolumn{2}{|c|}{ KL-M } & \multicolumn{2}{|c|}{ MD } \\
\hline Gender & MAE & CS $(\%)$ & MAE & CS $(\%)$ & MAE & CS $(\%)$ & MAE & CS (\%) \\
\hline Male & 6.99 & 55.26 & 4.89 & 64.14 & 4.58 & 68.25 & 4.46 & 69.23 \\
\hline Female & 8.16 & 50.45 & 5.96 & 57.22 & 5.86 & 58.48 & 5.80 & $\mathbf{5 8 . 5 3}$ \\
\hline
\end{tabular}

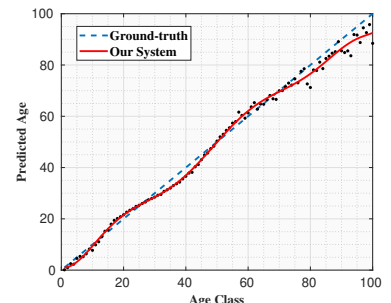

(a)

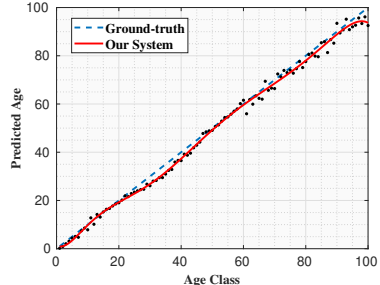

(b)
Figure 2. Average predicted age at each age class for each gender. (a) Male. (b) Female.

Table 2. MAEs and CS Scores with Respect to ethnicity

\begin{tabular}{|c|c|c|c|c|c|c|c|c|}
\hline & \multicolumn{2}{|c|}{ Microsoft } & \multicolumn{2}{|c|}{ CE-MV } & \multicolumn{2}{|c|}{ KL-M } & \multicolumn{2}{|c|}{ MD } \\
\hline Ethnicity & MAE & CS (\%) & MAE & CS (\%) & MAE & CS (\%) & MAE & CS (\%) \\
\hline European & 6.59 & 46.22 & 4.59 & 68.44 & 4.52 & 69.70 & 4.60 & 68.64 \\
\hline African & 7.21 & 42.32 & 5.45 & 58.92 & 5.12 & 63.19 & 4.96 & 64.80 \\
\hline Indian & 8.40 & 36.89 & 7.60 & 46.35 & 7.18 & 49.04 & 6.95 & 48.91 \\
\hline Chinese & 10.12 & 33.56 & 8.56 & 43.35 & 7.94 & 47.02 & 7.50 & 47.81 \\
\hline
\end{tabular}

\subsection{Effect of Ethnicity on Age Estimation}

To study the effect of ethnicity on the age estimation, we split the MORPH database into its dominant ethnicities, i.e., African and European. To include subjects from Indian and Chinese ethnicities we investigate two other databases, namely EFAD [23] and IFAD [29] respectively. Table 2 compares four age estimation methods using MAEs and CS scores for different ethnicities. Compared to other methods our age estimation system achieves better overall performance. Further, Fig. 3 illustrates the performance of our age estimation algorithm for different ethnicities at different age classes. The solid and dashed lines indicates the average predicted age and true age in each age class respectively. These results indicate that the age perception of European people is more accurate, compared to African and Asian people.

Our BAG training database is strongly imbalanced in terms of ethnicity with a large part consisting of European subjects. There are not enough images from Asian and African subjects at all age classes. For this reason, Chinese and Indian subjects, have the highest real age estimation er-
Table 3. MAEs and CS Scores with Respect to Expression

\begin{tabular}{|l|cc|cc|cc|cc|}
\hline & \multicolumn{2}{|c|}{ Microsoft } & \multicolumn{2}{c|}{ CE-MV } & \multicolumn{2}{c|}{ KL-M } & \multicolumn{2}{c|}{ MD } \\
\hline \hline Expression & MAE & CS (\%) & MAE & CS (\%) & MAE & CS (\%) & MAE & CS (\%) \\
\hline Neutrality & 5.57 & 70.25 & 3.56 & 80.09 & 3.46 & 79.84 & $\mathbf{3 . 2 6}$ & $\mathbf{8 0 . 1 0}$ \\
Sadness & 6.86 & 56.41 & 4.66 & $\mathbf{7 0 . 4 1}$ & $\mathbf{4 . 5 5}$ & 68.09 & 4.63 & 68.97 \\
Disgust & 7.15 & 53.85 & 5.17 & 63.99 & $\mathbf{4 . 7 4}$ & $\mathbf{6 6 . 9 6}$ & 4.98 & 63.72 \\
Fear & 7.55 & 53.99 & 5.35 & 63.95 & $\mathbf{5 . 2 2}$ & $\mathbf{6 4 . 2 6}$ & 5.54 & 59.59 \\
Anger & 6.95 & 56.81 & 4.80 & 66.94 & 4.58 & $\mathbf{6 9 . 7 0}$ & $\mathbf{4 . 5 3}$ & $\mathbf{6 9 . 8 5}$ \\
Happiness & 6.20 & 63.55 & 4.20 & 73.94 & $\mathbf{3 . 8 2}$ & $\mathbf{7 7 . 1 5}$ & 3.92 & 76.61 \\
\hline
\end{tabular}

ror, followed by African people. Interestingly, as can be observed from Fig. 3, the age of Chinese subjects is underestimated at all age ages, which may reflect some physiological phenomena. In fact, ethnicity and skin texture are related to each other. Skin texture of people of a certain ethnicity has many distinct facial characteristics that make its ageing process unique. Therefore, a model trained with images of a certain ethnicity cannot provide an accurate estimation of age of people with different ethnicity.

Let us focus only on the graphs related to European and African subjects in the most populated age ranges, i.e., from 20 to 70 years old, where the BAG database has enough images from these populations. A tendency to underestimate the age appears for younger people, being more pronounced for African people, while a tendency to overestimate age for older people appears. In general, our age estimation system tends to underestimate the ages, independently from ethnicity and gender attributes. Moreover, the underestimationto-overestimation point shifts from 40 years old for European people to 50 years old for African people. This suggests that each ethnicity has a different face ageing pattern, which should be taken into consideration during training of the model.

\subsection{Effect of Expression on Age Estimation}

To study the effect of expressions on age estimation, we evaluate different age estimation systems on FACES database which has 6 different expressions, i.e., neutrality, sadness, disgust, fear, anger, and happiness for each subject. Table 3 compares different age estimation methods in term of MAEs and CS scores under the same expression. Our age estimation method achieves higher performance than state-of-the-art algorithms for the neutral and angry faces, while it has lower accuracy with other expressions. The MAE value of our proposed method for different expressions ranging from 3.92 for neutral faces to 5.54 for fear expression shows the high sensitivity of our age estimation 


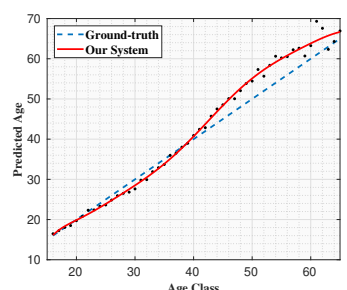

(a)

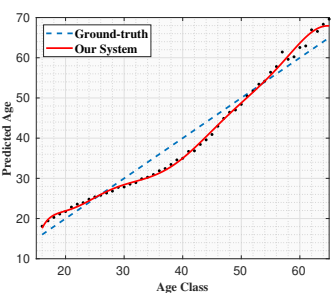

(b)

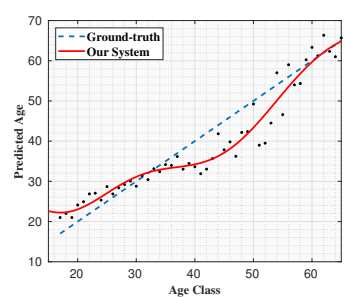

(c)

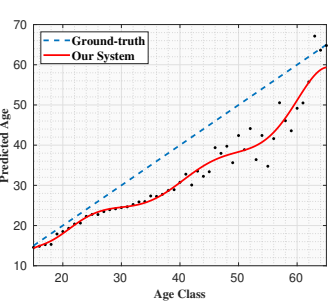

(d)

Figure 3. Average predicted age at each age class for different ethnicity. (a) European. (b) African. (c) Indian. (d) Chinese.

algorithm for facial expressions. This behaviour might be due to the fact that sagging of the face in any kind of expressions can resemble the effects of ageing. For example, some expressions form wrinkles over the face which can confuse the age estimation system to recognise the face as an older person. To make the age estimation system robust to diverse expressions, the age-related information across different facial expressions should be exploited during training.

\subsection{Effect of Image Quality on Age Estimation}

The input of an age estimation system should be provided by an imaging system which captures the ageing patterns such as, wrinkles and skin texture, faithfully. However, in real-world applications, it is often difficult to capture high quality face images of the observed individuals. Images are often taken by different imaging devices and may vary in quality and resolution. Therefore, it is important to study how the quality of capturing devices and distance of subjects to the camera influence the age estimation performance. To analyse the influence of the image quality on the age estimation performance, we perform experiments on the SC-FACE-SUR database which provides different images per each subject captured by five different quality surveillance cameras, named cam1-5, in day time, one infrared camera, named cam6 in night mode (NM) and one high resolution (HR) professional camera, named cam7. Further, the images were captured at three distances $(1.00 \mathrm{~m}$, $2.60 \mathrm{~m}$ and $4.20 \mathrm{~m}$ ) by cam $1-5$, providing images which differ substantially in quality and resolution. Images taken by these cameras have the following resolutions: $100 \times 75$ pixels for distance $3(4.20 \mathrm{~m}), 144 \times 108$ for distance $2(2.60 \mathrm{~m})$ and $224 \times 168$ for distance $1(1.00 \mathrm{~m})$. The images taken by cam 6 and cam 7 were captured in only one fixed distance and have $426 \times 320$ and 3, $072 \times 2,048$ pixels, respectively. It should be noted that cam5 is placed slightly above the head of the subject, who were not asked to look to a fixed point. This condition correspond to the scenario happens where the images are recorded by a surveillance camera which makes the age estimation system more demanding. Generally, the SC-FACE-SUR database contains images of extremely low quality and resolution. Fig. 4 shows examples of images captured by different cameras at different distances (resolutions).

Tables 4, 5 and 6 show MAEs and CS scores for different resolutions, respectively. In each table, the results for images taken with different cameras at a fixed distance are reported. In Table 4, we also report the results for the images taken by a high quality infrared camera, i.e. cam6, and a high quality camera, i.e. cam7, both taken at a fixed distance as a reference. As expected, age estimation performance improves as the resolution of the image increases. For instance, the MAE values of our proposed method for images taken by cam 1 ranges from 3.50 for resolution $224 \times 168$ (distance 1) to 5.44 for resolution $100 \times 75$ (distance 3). In conclusion, the resolution of face images, which is a function of the distance of the subject from the camera, has a strong impact on the age estimation performance.

As another interesting result, we can see the age estimation performance significantly depends on the quality of images captured by different cameras. According to the results in Table 4, the accuracy of the trained models for the images taken by surveillance cameras, i.e. cam1-5, is significantly lower than that for the image taken by the high quality camera, i.e. cam7. For instance, as can be seen from Table 4, the MAE values of our proposed method ranges from 3.40 for images taken by cam 2 to 4.09 for images captured by cam5. As mentioned before, cam5 is placed slightly above the head of a subject. Therefore, the face images captured at this distance show more of the top part of head. Different viewing angles to camera lead to self-occlusion. Therefore, as can be observed in Table 4, the accuracy for faces captured by cam 5 at distance 1 is significantly lower than that for images form other cameras. Finally, by comparing the results for cam6 in Table 4, we see that shooting in the night mode (NM) degrades the age estimation accuracy.

As a general conclusion, acquiring images with high quality has a significant impact on the accuracy of age estimation systems. Any change in the imaging conditions which distorts some ageing cues in the face, such as skin texture and wrinkles, has a negative influence on the accuracy. Designing a system which is robust to these imaging conditions can significantly improve the overall system performance. 


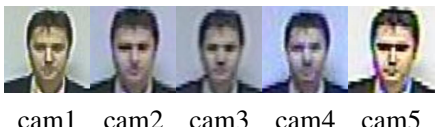

Distance $1=4.20 \mathrm{~m}$

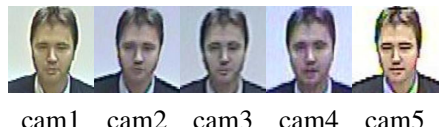

Distance $2=2.60 \mathrm{~m}$

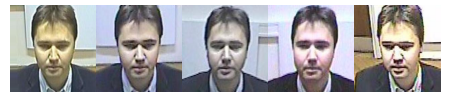

cam1 cam2 cam3 cam4 cam5

Distance $3=1.00 \mathrm{~m}$

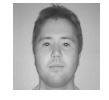

cam6

cam7

Figure 4. Face images of one individual in the SC-FACE-SUR database captured by five different quality surveillance cameras at three distances (cam1-5) and one infrared camera, cam6 in night mode (NM), and one high resolution (HR) professional camera, cam7.

Table 4. MAEs and CS Scores for Images captured by Cameras with Different Quality (Distance $1=1 \mathrm{~m}$ )

\begin{tabular}{|c|c|c|c|c|c|c|c|c|}
\hline & \multicolumn{2}{|c|}{ Microsoft } & \multicolumn{2}{|c|}{ CE-MV } & \multicolumn{2}{|c|}{ KL-M } & \multicolumn{2}{|c|}{ MD } \\
\hline Camera & MAE & CS (\%) & MAE & CS (\%) & MAE & CS (\%) & MAE & CS (\%) \\
\hline cam1 & 5.71 & 67.41 & 3.63 & 78.46 & 3.59 & 79.23 & 3.50 & 80.62 \\
\hline cam2 & 5.99 & 66.84 & 3.83 & 77.69 & 3.49 & 80.31 & 3.40 & 81.54 \\
\hline $\operatorname{cam} 3$ & 6.12 & 65.63 & 4.02 & 76.92 & 3.80 & 78.46 & 3.73 & 79.15 \\
\hline cam4 & 6.49 & 62.42 & 4.35 & 73.40 & 4.00 & 75.38 & 3.93 & 76.31 \\
\hline cam5 & 6.86 & 61.94 & 4.49 & 71.54 & 4.18 & 73.08 & 4.09 & 74.15 \\
\hline $\operatorname{cam6}(\overline{\mathbf{N}} \overline{\mathrm{M}})$ & $\overline{7} .3 \overline{2}$ & $5 \overline{6} .1 \overline{5}$ & 5.11 & 67.05 & $4 . \overline{3} 0$ & $\overline{7} 1 . \overline{5} 4$ & $4 . \overline{28}$ & $\overline{71} . \overline{7} 7$ \\
\hline cam7 (HR) & 4.24 & 76.79 & 2.71 & 87.69 & 2.56 & 91.54 & 2.50 & 91.54 \\
\hline
\end{tabular}

Table 5. MAEs and CS Scores for Images captured by Cameras with Different Quality (Distance 2 $=2.60 \mathrm{~m}$ )

\begin{tabular}{|c|c|c|c|c|c|c|c|c|}
\hline & \multicolumn{2}{|c|}{ Microsoft } & \multicolumn{2}{|c|}{ CE-MV } & \multicolumn{2}{|c|}{ KL-M } & \multicolumn{2}{|c|}{ MD } \\
\hline Camera & MAE & CS (\%) & MAE & CS (\%) & MAE & CS (\%) & MAE & CS (\%) \\
\hline cam1 & 7.68 & 57.89 & 5.58 & 68.77 & 5.15 & 70.00 & 5.06 & 70.85 \\
\hline cam2 & 7.61 & 57.75 & 5.51 & 68.98 & 5.10 & 70.62 & 5.00 & 70.92 \\
\hline cam3 & 7.85 & 57.15 & 5.65 & 68.07 & 5.53 & 68.62 & 5.25 & 69.23 \\
\hline cam4 & 8.12 & 56.81 & 5.90 & 67.77 & 5.60 & 68.08 & 5.32 & 68.78 \\
\hline cam5 & 8.13 & 56.95 & 5.99 & 67.30 & 5.63 & 67.85 & 5.48 & 67.57 \\
\hline
\end{tabular}

Table 6. MAEs and CS Scores for Images captured by Cameras with Different Quality (Distance 3=4.20m)

\begin{tabular}{|c|c|c|c|c|c|c|c|c|}
\hline & \multicolumn{2}{|c|}{ Microsoft } & \multicolumn{2}{|c|}{ CE-MV } & \multicolumn{2}{|c|}{ KL-M } & \multicolumn{2}{|c|}{ MD } \\
\hline Camera & MAE & CS (\%) & MAE & CS (\%) & MAE & CS (\%) & MAE & CS (\%) \\
\hline cam1 & 7.50 & 54.12 & 5.40 & 65.02 & 5.45 & 64.62 & 5.44 & 64.69 \\
\hline $\operatorname{cam} 2$ & 7.83 & 53.13 & 5.82 & 64.00 & 5.75 & 64.40 & 5.73 & 64.46 \\
\hline $\operatorname{cam} 3$ & 8.96 & 50.73 & 6.76 & 61.70 & 6.91 & 61.54 & 6.30 & 62.08 \\
\hline cam4 & 8.61 & 50.95 & 6.55 & 61.82 & 6.36 & 62.01 & 6.47 & 61.92 \\
\hline cam5 & 8.23 & 52.63 & 6.00 & 63.62 & 5.90 & 64.34 & 5.91 & 64.23 \\
\hline
\end{tabular}

\subsection{Effect of Head Pose on Age Estimation}

To evaluate the age estimation performance in terms of pose variation, we show the MAE values and CS scores in Table 7 for all poses in the SC-FACE-ROT database, compared with different age estimation algorithms. As expected, the frontal images provide better age estimation performance among a large range of pose variations ranging from -90 (left profile) to +90 (right profile) degrees in equal steps of 22.5 degrees. We observe a gradual reduction in the age estimation performance if the head pose moves away from the frontal pose. This is more pronounced for semi-left profiles. Note that the accuracy drops significantly for even near frontal profiles. These results are very infor-
Table 7. MAEs and CS Scores for All Poses in the SC-FACE-ROT Database

\begin{tabular}{|l|cc|cc|cc|cc|}
\hline & \multicolumn{2}{|c|}{ Microsoft } & \multicolumn{2}{c|}{ CE-MV } & \multicolumn{2}{c|}{ KL-M } & \multicolumn{2}{c|}{ MD } \\
\hline \hline Degree & MAE & CS (\%) & MAE & CS (\%) & MAE & CS (\%) & MAE & CS (\%) \\
\hline $\mathbf{- 9 0}$ & 8.83 & 32.08 & 6.81 & 43.08 & $\mathbf{7 . 4 9}$ & $\mathbf{4 9 . 2 3}$ & 8.30 & 36.92 \\
$\mathbf{- 6 7 . 5}$ & 7.15 & 49.77 & 5.05 & 60.77 & 5.50 & 53.08 & $\mathbf{4 . 9 2}$ & $\mathbf{6 8 . 4 6}$ \\
$\mathbf{- 4 5}$ & 5.47 & 72.81 & 3.48 & 83.85 & 4.06 & 70.00 & $\mathbf{3 . 4 3}$ & $\mathbf{8 3 . 8 5}$ \\
$\mathbf{- 2 2 . 5}$ & 5.65 & 66.60 & 3.50 & 77.68 & 2.93 & 89.23 & $\mathbf{2 . 8 5}$ & $\mathbf{9 0 . 0 0}$ \\
Profile & 4.63 & 76.72 & 2.71 & 87.69 & 2.56 & 91.54 & $\mathbf{2 . 5 0}$ & $\mathbf{9 1 . 5 4}$ \\
$\mathbf{+ 2 2 . 5}$ & 5.65 & 69.13 & 3.71 & 80.00 & 3.70 & 80.00 & $\mathbf{3 . 3 0}$ & $\mathbf{8 6 . 1 5}$ \\
$\mathbf{+ 4 5}$ & 6.71 & 44.44 & 4.85 & 56.92 & 5.35 & 59.23 & $\mathbf{4 . 5 2}$ & $\mathbf{7 2 . 3 1}$ \\
$\mathbf{+ 6 7 . 5}$ & 8.49 & 37.86 & 6.41 & 48.46 & 7.23 & 46.15 & $\mathbf{5 . 8 7}$ & $\mathbf{5 6 . 9 2}$ \\
$\mathbf{+ 9 0}$ & 10.08 & 28.45 & 7.50 & 40.77 & 8.35 & 43.85 & $\mathbf{6 . 8 3}$ & $\mathbf{5 4 . 6 2}$ \\
\hline
\end{tabular}

mative for the practical real age estimation. In conclusion, the impact of pose should be taken into consideration for an accurate age estimation algorithm.

\subsection{Effect of Illumination Conditions}

Illumination variation is one of many sources of variations that can have a strong effect on the performance of age estimation. In fact, changes in illumination can make the predicted age of the same person to vary significantly. To study the effects of changes in illumination, we use the MULTI-PIE database. Recall that the MULTI-PIE database has 24 images per each subject with 24 different illumination conditions. In this database, changes in illumination are systematically varied, as shown in Fig. 5. The database provides one high resolution image for each subject as well. Since the age label of the subjects are not available, we consider the estimated age from this high resolution image as the reference age. Fig. 6 shows the MAE values at each illumination variant for different age estimation algorithms. The horizontal axis indicates the index associated with the illumination variant according to Fig. 5. As can be observed, there is a dramatic fluctuation in the predicted age associated with changes in illumination. These results reveal that the lighting condition should be accounted for when training the model.

\subsection{Effect of Makeup on Age Estimation}

We crawled a database, consisting of 200 subjects, from Internet. There are two images per each subject; one image before and another one after the application of makeup. Fig. 7 illustrates the performance of our age estimation al- 


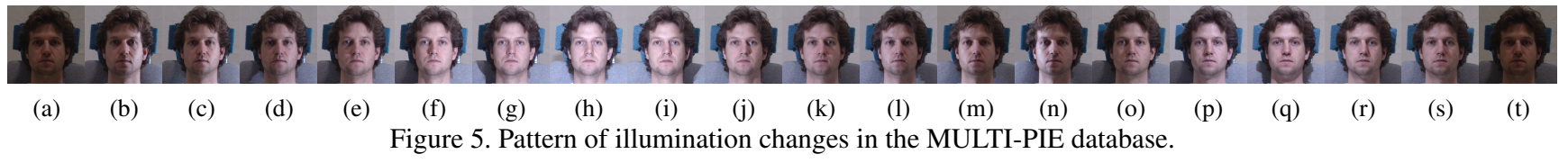

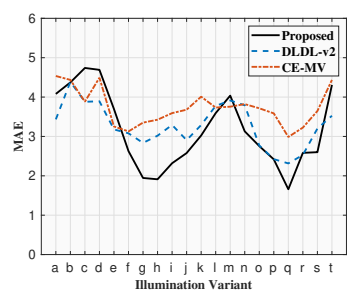

Figure 6. Effect of illumination.

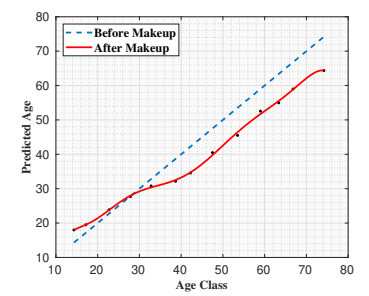

Figure 7. Effect of makeup.

gorithm for different age classes. The solid line indicates the average predicted age as a function of true age after makeup. The dashed line refers to the average predicted age before makeup. As can be observed, for all subjects more than 27 years old, we notice a constant underestimation of the real age. This trend is more pronounced for ages above 60 . The reason for this behaviour is the masking effect of makeup which leads to hiding the ageing signs, such as wrinkles. Interestingly, as can be observed from Fig. 7, the makeup application leads to an overestimation of age for subjects younger than 27 years old. In conclusion, makeup application makes younger women look older but makes older women look younger. One possible answer to this fact is, in addition to hiding the ageing patterns by makeup, it also accentuates some visual features of the face which might be associated with the ageing patterns used by our system to predict the real age. This kind of flattening of the age range with makeup is should be taken into account to improve the robustness of the age estimation system in the future.

\subsection{Discussion: Characteristics of BAG Database}

It should be noted that we have no information about the distribution of intrinsic and extrinsic factors characterising the images in the BAG database. Moreover, there is no information about the attribute of images in terms of illumination, pose, quality, etc. All the knowledge we have about the BAG database is that the numbers of male and white subjects are greater than numbers of female and black subjects, respectively. There are a few Asian subjects as well. Annotating the BAG database with all these factors would be a cumbersome and expensive task. However, our focus in this study is not to find out which of these factors can be improved with more representative training data. Obviously, the performance will be improved by diversifying of the training data. In fact, improving the training database with samples which are representative of different factors

is not always practical for ageing databases, where data is relatively scarce and expensive to generate.

Our focus in this paper is on how to effectively utilise the existing training databases, regardless of the actual distribution of training data, either to identify which algorithms are more robust to these factors, or to qualify the caveat for the usability of the estimates. In this context, we compare different age estimation systems in terms of their robustness to various internal and external factors. As part of the exercise, we experimentally demonstrate that some loss functions achieve better model generalisation than others.

\section{Conclusion}

We conducted a sensitivity analysis to gain insight and understanding of the impact of subject-, camera- and environmental factors on the quality and performance of an age estimation system. The results showed that the performance of all age estimation systems were significantly affected by these factors. The image quality and illumination conditions, in particular, have a notable effect on the performance of existing age estimation systems. Our evaluation results emphasise that the demographic factors, such as gender and ethnicity, have an impact on the overall accuracy of all age prediction models. Expression and the application of makeup and self-occlusion have also been found to influence the accuracy of all four considered systems. Apart from raising the community awareness about these sensitivities, it is hoped that the study will help to motivate and target future research in the face based age estimation area.

\section{Acknowledgement}

This work was supported in part by the EPSRC Programme Grant (FACER2VM) EP/N007743/1 and the EPSRC/dstl/MURI project EP/R018456/1.

\section{References}

[1] A. Akbari and M. Trocan. Downsampling based image coding using dual dictionary learning and sparse representations. In International Workshop on Multimedia Signal Processing (MMSP), pages 1-5, 2018.

[2] A. Akbari, M. Trocan, and B. Granado. Image error concealment using sparse representations over a trained dictionary. In Picture Coding Symposium (PCS), pages 1-5, 2016.

[3] A. Akbari, M. Trocan, and B. Granado. Image error concealment based on joint sparse representation and non-local 
similarity. In IEEE Global Conference on Signal and Information Processing (GlobalSIP), pages 6-10, 2017.

[4] A. Akbari, M. Trocan, and B. Granado. Joint-domain dictionary learning-based error concealment using common space mapping. In International Conference on Digital Signal Processing (DSP), pages 1-5, 2017.

[5] A. Akbari, M. Trocan, and B. Granado. Sparse recoverybased error concealment. IEEE Transactions on Multimedia, 19(6):1339-1350, 2017.

[6] A. Akbari, M. Trocan, S. Sanei, and B. Granado. Joint sparse learning with nonlocal and local image priors for image error concealment. IEEE Transactions on Circuits and Systems for Video Technology, 30(8):2559-2574, 2020.

[7] F. Anda, D. Lillis, N. Le-Khac, and M. Scanlon. Evaluating automated facial age estimation techniques for digital forensics. In IEEE Security and Privacy Workshops (SPW), pages 129-139, May 2018.

[8] M. Bashar, A. Akbari, K. Cumanan, H. Q. Ngo, A. G. Burr, P. Xiao, and M. Debbah. Deep learning-aided finite-capacity fronthaul cell-free massive mimo with zero forcing. In IEEE International Conference on Communications (ICC), pages 1-6, 2020.

[9] M. Bashar, A. Akbari, K. Cumanan, H. Q. Ngo, A. G. Burr, P. Xiao, M. Debbah, and J. Kittler. Exploiting deep learning in limited-fronthaul cell-free massive mimo uplink. IEEE Journal on Selected Areas in Communications (JSAC), 38(8):1678-1697, 2020.

[10] S.-H. Cha. Comprehensive survey on distance/similarity measures between probability density functions. International Journal of Mathematical Models and Methods in Applied Sciences, 1(1):300-307, Nov 2007.

[11] A. Clapés, G. Anbarjafari, O. Bilici, D. Temirova, E. Avots, and S. Escalera. From apparent to real age: Gender, age, ethnic, makeup, and expression bias analysis in real age estimation. In IEEE Conference on Computer Vision and Pattern Recognition Workshops (CVPRW), pages 2436-243609, June 2018

[12] N. C. Ebner, M. Riediger, and U. Lindenberger. Faces-a database of facial expressions in young, middle-aged, and older women and men: Development and validation. Behavior Research Methods, 42(1):351-362, Feb 2010.

[13] B. Gao, C. Xing, C. Xie, J. Wu, and X. Geng. Deep label distribution learning with label ambiguity. IEEE Transactions on Image Processing, 26(6):2825-2838, June 2017.

[14] B.-B. Gao, H.-Y. Zhou, J. Wu, and X. Geng. Age estimation using expectation of label distribution learning. In Proceedings of International Joint Conference on Artificial Intelligence (IJCAI), pages 712-718, 72018.

[15] X. Geng. Label distribution learning. IEEE Transactions on Knowledge and Data Engineering, 28(7):1734-1748, July 2016.

[16] X. Geng, C. Yin, and Z. Zhou. Facial age estimation by learning from label distributions. IEEE Transactions on Pattern Analysis and Machine Intelligence, 35(10):2401-2412, Oct 2013.

[17] M. Grgic, K. Delac, and S. Grgic. Scface - surveillance cameras face database. Multimedia Tools and Applications, 51(3):863-879, Feb 2011.
[18] R. Gross, I. Matthews, J. Cohn, T. Kanade, and S. Baker. Multi-pie. In IEEE International Conference on Automatic Face Gesture Recognition, pages 1-8, Sep 2008.

[19] Y. Lee, J. J. Filliben, R. J. Micheals, and P. J. Phillips. Sensitivity analysis for biometric systems: A methodology based on orthogonal experiment designs. Computer Vision and Image Understanding, 117(5):532 - 550, 2013.

[20] W. Li, J. Lu, J. Feng, C. Xu, J. Zhou, and Q. Tian. Bridgenet: A continuity-aware probabilistic network for age estimation. In IEEE Conference on Computer Vision and Pattern Recognition (CVPR), June 2019.

[21] S. Moschoglou, A. Papaioannou, C. Sagonas, J. Deng, I. Kotsia, and S. Zafeiriou. Agedb: The first manually collected, in-the-wild age database. In IEEE Conference on Computer Vision and Pattern Recognition Workshops (CVPRW), pages 1997-2005, July 2017.

[22] M. Ngan and P. Grother. Face recognition vendor test (frvt), performance of automated age estimation algorithms. Technical Report 7995, National Institute of Standards and Technology, Gaithersburg, MD, US, Mar 2014.

[23] Z. Niu, M. Zhou, L. Wang, X. Gao, and G. Hua. Ordinal regression with multiple output cnn for age estimation. In IEEE Conference on Computer Vision and Pattern Recognition (CVPR), pages 4920-4928, June 2016.

[24] H. Pan, H. Han, S. Shan, and X. Chen. Mean-variance loss for deep age estimation from a face. In IEEE Conference on Computer Vision and Pattern Recognition, pages 52855294, June 2018.

[25] G. Panis, A. Lanitis, N. Tsapatsoulis, and T. F. Cootes. Overview of research on facial ageing using the fg-net ageing database. IET Biometrics, 5(2):37-46, May 2016.

[26] O. M. Parkhi, A. Vedaldi, and A. Zisserman. Deep face recognition. In British Machine Vision Conference, 2015.

[27] K. Ricanek and T. Tesafaye. Morph: a longitudinal image database of normal adult age-progression. In International Conference on Automatic Face and Gesture Recognition (FGR), pages 341-345, Apr 2006.

[28] R. Rothe, R. Timofte, and L. Van Gool. Deep expectation of real and apparent age from a single image without facial landmarks. International Journal of Computer Vision, 126(2):144-157, Apr 2018.

[29] R. Sharma and M. Patterh. Indian face age database: A database for face recognition with age variation. International Journal of Computer Applications, 126(5):21-27, Sep 2015.

[30] Y. Wang, X. Ma, Z. Chen, Y. Luo, J. Yi, and J. Bailey. Symmetric cross entropy for robust learning with noisy labels. In 2019 IEEE/CVF International Conference on Computer Vision (ICCV), pages 322-330, 2019.

[31] K. Zhang, C. Gao, L. Guo, M. Sun, X. Yuan, T. X. Han, Z. Zhao, and B. Li. Age group and gender estimation in the wild with deep ror architecture. IEEE Access, 5:2249222503, 2017.

[32] Z. Zhang, Y. Song, and H. Qi. Age progression/regression by conditional adversarial autoencoder. In IEEE Conference on Computer Vision and Pattern Recognition (CVPR), pages 4352-4360, July 2017. 